\title{
Atherosclerotic Lesion of the Carotid Artery in Indonesian Cynomolgus Monkeys Receiving a Locally Sourced Atherogenic Diet
}

\author{
Sri Rahmatul Laila 1,*(D), Dewi Apri Astuti ${ }^{2,3}$, Irma Herawati Suparto ${ }^{3,4}$, Ekowati Handharyani ${ }^{3,5}$, \\ Thomas C. Register ${ }^{6}$ and Dondin Sajuthi ${ }^{3,5}$
}

check for

updates

Citation: Laila, S.R.; Astuti, D.A.; Suparto, I.H.; Handharyani, E.; Register, T.C.; Sajuthi, D. Atherosclerotic Lesion of the Carotid Artery in Indonesian Cynomolgus Monkeys Receiving a Locally Sourced Atherogenic Diet. Vet. Sci. 2022, 9, 105. https://doi.org/10.3390/ vetsci9030105

Academic Editor: Emrah Yatkin

Received: 27 December 2021

Accepted: 24 February 2022

Published: 26 February 2022

Publisher's Note: MDPI stays neutral with regard to jurisdictional claims in published maps and institutional affiliations.

Copyright: (C) 2022 by the authors. Licensee MDPI, Basel, Switzerland. This article is an open access article distributed under the terms and conditions of the Creative Commons Attribution (CC BY) license (https:// creativecommons.org/licenses/by/ $4.0 /)$.
1 Departement of Anatomy, Physiology, and Pharmacology, Faculty of Veterinary Medicine, IPB University, Bogor 16680, Indonesia

2 Departement of Nutrition and Feed Technology, Faculty of Animal Science, IPB University, Bogor 16680, Indonesia; dewiaa@apps.ipb.ac.id

3 Primate Research Center, IPB University, Bogor 16680, Indonesia; irmasu@apps.ipb.ac.id (I.H.S.); ekowatieko@apps.ipb.ac.id (E.H.); dondinsa@apps.ipb.ac.id (D.S.)

4 Departement of Chemistry, Faculty of Mathematics and Natural Sciences, IPB University, Bogor 16680, Indonesia

5 Departement of Veterinary Clinic, Reproduction, and Pathology, Faculty of Veterinary Medicine, IPB University, Bogor 16680, Indonesia

6 Centre for Comparative Medicine Research, Wake Forest School of Medicine, Winston-Salem, NC 27157, USA; register@wakehealth.edu

* Correspondence: srirlaila@apps.ipb.ac.id

\begin{abstract}
The atherosclerotic lesion is a principal hallmark of atherosclerotic animal models. This study aimed to assess lesions of the carotid artery in Indonesian cynomolgus monkeys exposed to an IPB-1 atherogenic diet. A total of 20 adult male cynomolgus monkeys received the local IPB-1 diet for two years. Blood lipid profiles, morphology, and carotid ultrasound of monkeys were measured. Nine of them were euthanized to confirm atherosclerotic lesions. Common carotid arteries (CCA) and carotid bifurcation (BIF) samples were collected and stained using Verhoef-van Giessen and CD68 immunohistochemistry. The results reveal the presence of severe atherosclerosis plaques in six out of nine animals $(66.7 \%)$ corresponding to intermediately and hyper-responsive groups. The hyper-responsive group displayed the highest response in the developing intimal area (IA) at the CCA $\left(0.821 \mathrm{~mm}^{2}\right)$, whereas the hyporesponsive group had the smallest IA $\left(0.045 \mathrm{~mm}^{2}\right)(p=0.0001)$. At the BIF, the hyporesponsive group showed the smallest IA $(p=0.001)$, but there was no difference between the intermediately and hyper-responsive groups $(p=0.312)$. The macrophage marker CD68 was also expressed on the cartotid of the intermediately and hyper-responsive groups. These results indicate that severe atherosclerotic lesions with high infiltration of macrophages were formed in the carotid arteries of intermediately and hyper-responsive Indonesian cynomolgus monkeys fed with the local atherogenic diet IPB-1 over two years, thus confirming atherosclerosis in a nonhuman primate model.
\end{abstract}

Keywords: atherosclerosis; carotid artery; CD68; cynomolgus monkey; lesions; diet

\section{Introduction}

The cynomolgus monkey (Macaca fascicularis) is the non-human primate most used in biomedical research after the rhesus monkey (Macaca mulatta). Indonesian cynomolgus monkeys are exported primarily to the United States [1] for biomedical research pertaining to infectious diseases as well as degenerative diseases [2]. The Indonesian cynomolgus monkey ( $M$. fascicularis fascicularis) has a unique genetic profile, which makes it particularly suitable for biomedical research [3]. Cynomolgus is used in biomedical research, with many advantages including anatomical and physiological aspects that are similar to humans, 
such as in cardiovascular disease [4]. In atherosclerosis, cynomolgus can be used to study the mechanisms of pathophysiology and the formation of lesions, up to the appearance of clinical symptoms due to such lesions [5]. However, the International Union for Conservation of Nature (IUCN) has reported that the cynomolgus population has significantly decreased, making its status vulnerable [6], which raises awareness regarding the use of cynomolgus monkeys in research.

In the last decade, our team at the Bogor Primate Research Center (PRC)-IPB University, Indonesia, has lead an initiative promoting biomedical research with cynomolgus monkeys nationally to reduce exportation and provide early treatment prior to advance studies abroad. This initiative resulted in the development of a local diet for atherosclerosis studies named the Institut Pertanian Bogor-1 (IPB-1) atherogenic diet. This diet had good digestibility value and is well absorbed, allowing the maintenance of normal body weight and adipose index of the monkeys [7]. This diet can also be used for long-term feeding without inducing major complications in animals [8]. In a previous study, the IPB-1 atherogenic diet was able to induce hypercholesterolemia and increase the intima-media thickness surrounding the carotid artery, as clinically observed by ultrasound, in cynomolgus monkeys [9]. These results support conducting clinical research on atherosclerosis lesions without euthanizing the monkeys. However, further observations characterizing the formation of atherosclerotic lesions in the carotid artery are warranted for confirmation that cynomolgus monkeys receiving the IPB-1 diet represent an excellent model for investigating human atherosclerotic lesions of the artery.

The atherosclerotic lesion is one of the main parameters that are still widely used for evaluating atherosclerosis in animal models. As an indicator of atherogenesis, cellular and biochemical components can be detected by histological techniques. The initiation process of atherosclerosis involves the trapping of low-density lipoprotein (LDL) in the intimal matrix and its oxidation, together with the stimulation of inflammatory and immune responses that are associated with intimal migration and/or proliferation of particular cell types, predominantly macrophages $[10,11]$. The development of an atherosclerosis lesion begins with endothelial dysfunction, a fatty streak, atheroma, followed by a fibrous cap atheroma, and finally complex plaques [12]. Previous studies, which were mainly conducted in the United States using a "Western diet", have reported atherosclerotic lesions in cynomolgus monkeys since the 1970s [13-16]. The IPB-1 atherogenic diet differs from the Western diet in terms of composition and nutrition, making it relevant to thoroughly test its outcomes on the atherosclerotic lesion, which is key to developing an Indonesian monkey model for atherosclerosis. Our previous findings demonstrate 3 response groups including the slightly elevated cholesterol in the plasma of monkeys (intermediate responsive group) [9], while Clarkson et al. only observed this for the hypo- and hyper-responsive groups [14]. It is interesting to note that atherosclerotic lesions in the carotid artery of individuals in the intermediately responsive group would be the same as those in the hyper-responsive group. In this study, we evaluated the grade of the plaques (American Heart Association/AHA grade) $[17,18]$ and a cellular marker of macrophages involved in atherogenesis [19]. The establishment of the IPB-1-diet-induced monkey model could help researchers to perform studies on the prevention, detection, and treatment of atherosclerosis diseases in their country of origin.

\section{Materials and Methods}

\subsection{Experimental Design}

We used a locally sourced diet, the IPB-1 atherogenic diet, produced at PRC-IPB, Indonesia. This diet contains egg yolk as a cholesterol source, coconut oil and beef tallow as a saturated fatty acid, and corn oil as a polyunsaturated fatty acid (PUFA) [7]. The macronutrient differences between the IPB-1 atherogenic diet and the Western diet formula for the cynomolgus model [14] are shown in Table 1. All procedures were approved by the Animal Care and Use Committee from PRC-IPB (Number PRC-14-B003). A total of 20 male cynomolgus monkeys (aged 6-7 years, weight 5-6 kg) were fed the IPB-1 atherogenic 
diet (containing $0.28 \mathrm{mg} / \mathrm{Cal}$ of cholesterol daily) for two years. We previously measured different atherosclerosis risk factors (total plasma cholesterol (TPC), LDL, high-density lipoprotein (HDL), triglyceride (TG), blood glucose (Glu), and body mass index (BMI)) in all of the monkeys included in the current study [9]. These monkeys showed different responses to the cholesterol content of the diet. The differential response of cynomolgus monkeys to dietary cholesterol was reported in another study previously in which monkeys were divided only into 2 groups, hyporesponsive, for TPC around $200 \mathrm{mg} / \mathrm{dL}$, and hyperresponsive, for TPC above $400 \mathrm{mg} / \mathrm{dL}$ [14]. In this current study, we observed monkeys with TPC between 200 and $400 \mathrm{mg} / \mathrm{dL}$, so the monkeys were divided into three groups: hyporesponsive (TPC $<200 \mathrm{mg} / \mathrm{dL}$ ), intermediately responsive (TPC 200-400 mg/dL), and hyper-responsive (TPC $>400 \mathrm{mg} / \mathrm{dL}$ ) groups, corresponding to four, eight, and eight monkeys in each group, respectively. For post-mortem investigation, we euthanized nine monkeys using a group randomized design, in which three monkeys represented each group (hypo-, intermediately, and hyper-responsive).

Table 1. Differences between IPB-1 atherogenic diet and Western diet $[7,9,14,16]$.

\begin{tabular}{|c|c|c|}
\hline Macronutrient & $\begin{array}{l}\text { Western Diet } \\
\text { (Sources/Level) }\end{array}$ & $\begin{array}{c}\text { IPB-1 Diet } \\
\text { (Sources/Level) }\end{array}$ \\
\hline Protein (animal) & Casein and lactalbumin: high & Fish meal: moderate \\
\hline Protein (plant) & Wheat flour: low & Soya meal: moderate \\
\hline Saturated fats & Butter: high & $\begin{array}{l}\text { Coconut oil and beef tallow: } \\
\text { moderate }\end{array}$ \\
\hline Unsaturated fats & Apple sauce: low & Corn oil: normal \\
\hline Carbohydrates & Sugar, wheat flour: high & Sugar, wheat flour: high \\
\hline Cholesterol & $\begin{array}{l}\text { Cholesterol: high } \\
(0.33-0.35 \mathrm{mg} \text { chol } / \mathrm{Cal})\end{array}$ & $\begin{array}{l}\text { Egg yolk: moderate } \\
(0.28-0.29 \mathrm{mg} \text { chol/Cal })\end{array}$ \\
\hline
\end{tabular}

chol $=$ cholesterol

\subsection{Necropsy Procedure}

The necropsy was performed by a certified pathologist following the procedure in the Laboratory of Pathology, PRC-IPB, Bogor. Ketamine $10 \%$ (Ket-A-100 ${ }^{\circledR}$, Senasa, Peru) $10 \mathrm{mg} / \mathrm{kg}$ of body weight (BW) and xylazine $2 \%$ (Dormi-Xyl ${ }^{\circledR} 20$, Senasa, Peru) $0.5 \mathrm{mg} / \mathrm{kg}$ of BW were administered intramuscularly as a sedative agent before necropsy. Afterwards, a low dose of sodium pentobarbital (Doléthal ${ }^{\circledR}$, Espanola) $(100 \mathrm{mg} / \mathrm{kg}$ ) was injected intravenously to prevent sudden death of the monkeys. The heart of the monkey was kept beating for the transcardial perfusion procedure. The circulatory system was flushed with $\mathrm{NaCl}$ saline $(0.9 \%)$ and perfused with $4 \%$ paraformaldehyde for $1 \mathrm{~h}$ under a pressure of $100 \mathrm{~mm} \mathrm{Hg}$ [20]. The common carotid arteries (CCA) and the carotid bifurcation (BIF) were collected at necropsy and post-fixed into $4 \%$ paraformaldehyde for about $48 \mathrm{~h}\left(4{ }^{\circ} \mathrm{C}\right)$. One block was taken from the BIF, while one every three blocks were taken from the right and left CCA (5 $\mathrm{mm}$ in length, cut perpendicular to the long axis of the artery) then stored at a specific tissue cassette in stopping point alcohol 70\%.

\subsection{Histological Preparation and Verhoef-Van Gieson (VVG) Staining}

All the selected artery blocks were dehydrated in graded alcohol (80, 90, 95, and $100 \%$ ), cleared with three changes of xylol, then infiltrated and embedded in paraffin (20). The paraffin blocks of carotid arteries were cut to $0.5 \mu \mathrm{m}$ thickness using a manual rotary microtome. From each block, two serial sections were mounted, one on a non-coated slide for VVG histochemical staining, and one on a poly-L-lysine coated slide for CD-68 /macrophages immunohistochemical (IHC) staining.

VVG staining was performed to identify elastic fibers of the carotid arteries, using a protocol provided by the pathology laboratory, Section for Comparative Medicine, Wake Forest University School of Medicine, Winston-Salem, NC, USA. The slides were first deparaffinized and hydrated with distilled water. They were subsequently stained in Verhoeff's solution for $1 \mathrm{~h}$, and then rinsed in tap water with 2-3 changes. Next, the slides 
were differentiated in $2 \%$ ferric chloride for 1-2 min then rinsed with tap water to stop the differentiation and examined microscopically (light microscope with $10 \times$ objective lens magnification) for black elastic fiber staining and gray background. The next step was to treat the slides with $5 \%$ sodium thiosulfate for $1 \mathrm{~min}$ followed by washing in running tap water for $5 \mathrm{~min}$. Furthermore, the slides were counterstained in Van Gieson's solution for 3-5 min, followed by dehydration through $95 \%$ alcohol and two changes of $100 \%$ alcohol quickly. Lastly, the slides were cleared with two changes of xylene for $3 \mathrm{~min}$ each and coverslipped. The VVG staining displayed stained elastic fibers blue-black to black, nuclei blue to black, collagen red, while other tissue elements or background were yellow on observation with a light microscope.

\subsection{Immunohistochemical Staining for $\mathrm{CD} 68$}

For immunohistochemical staining procedures, the slides were initially deparaffinized and hydrated as described above. Heat-induced epitope retrieval using citrate buffer was performed for antigen retrieval $(20 \mathrm{~min}$ at $\mathrm{pH}$ 6.0). Then, the slides were incubated with normal serum for $20 \mathrm{~min}\left(37^{\circ} \mathrm{C}\right)$ to block nonspecific staining. After blocking, the slides were incubated with monoclonal antibody mouse anti-human CD68 MCA5709, Clone KP-1 IgG (1:50) from BIO-RAD (Hercules, CA, USA) in $10 \mathrm{mM}$ sodium phosphatase, $\mathrm{pH}$ 7.2-7.4 for $30 \mathrm{~min}\left(37^{\circ} \mathrm{C}\right)$. Afterwards, the slides were incubated using the biotinylated Vectastain Universal ABC-AP kit AK-5200 for $30 \mathrm{~min}\left(37^{\circ} \mathrm{C}\right)$ as a secondary antibody. For visualization of the staining, the slides were incubated with Vector Red (Vector Labs, Burlingame, CA, USA) for $30 \mathrm{~min}\left(37^{\circ} \mathrm{C}\right)$. Tris-buffered saline was used as a washing solution in all the immunostaining steps mentioned above. Subsequently, the slides were incubated for 10-15 min in alkaline phosphatase substrate solution, and then washed with deionized water for $5 \mathrm{~min}$. Lastly, the slides were counterstained with Harris hematoxylin for 1-2 min, dehydrated, cleared, and mounted (Entellan, Merck Darmstadt, Germany) immediately. Positive CD68 staining was indicated by a red color, while negative CD68 staining was indicated by colorless areas. One minute hematoxylin was used as a counterstain, showing a blue color in the nuclei by means of a light microscope.

\subsection{Analysis of Plaque Severity and Extent}

Plaque severity and extent were measured from the VVG-stained slides. Plaque severity was determined using a well-established protocol for specifying a grade from I-III as initial lesions and IV to VI as an advanced lesion (American Heart Association Grade) $[15,16]$. The grade I lesions contain atherogenic lipoprotein (LDL) associated with an increase in macrophage infiltration and the formation of macrophages filled with lipid droplets (foam cells) in the arterial intima. This lesion usually occurred in arteries with adaptive thickening of the intima, which is normally very thin and represents adaptations to local mechanical forces. Grade II lesions consist of macrophage foam cells and smooth muscle cells containing lipid droplets designated as fatty streaks. Grade III lesions are characterized by pools of extracellular lipid droplets and particles in addition to all the components of grade II lesions. Grade IV lesions are characterized by a larger, united, and disruptive core of extracellular lipids known as the lipid core or atheroma. Grade V lesions have a lipid core, in which prominent new fibrous connective tissue has formed and is referred to as fibroatheroma. Some grade V lesions are also largely calcified. Lastly, grade VI lesions represent grade V lesions with disruptions of the lesion surface, hematoma, or hemorrhaging. This type may also be referred to as complicated lesions [18].

The plaque severity scores for each monkey were determined as the maximum severity grade in each CCA and BIF. We chose the maximum grade because the clinical event of cardiovascular disease, especially coronary artery disease, relates to its most complicated lesion [21]. Plaque extent was presented as the intimal area (IA), internal elastic lamina length (IELL), and maximum intimal thickness (MXIT) of the carotid artery. In each animal, the average of IELL, IA, and MXIT was calculated for both CCA and BIF. 


\subsection{Data and Statistical Analysis}

Plaque severity of the carotid artery was analyzed descriptively. The extent of the plaque (IA, IELL, and MXIT) was analyzed quantitatively using Image-Pro Plus Ver 9.1. (Media Cybernetics ${ }^{\circledR}$, Rockville, MD, USA) and reported as mean $+/-$ standard error. The CD68 immunostaining was analyzed quantitatively using Visio Pharm image analyses (VISIO PHARM $^{\circledR}$, Broomfield, CO, USA). One-way analysis of variance (ANOVA) (IBM SPSS) was used to assess parameter differences among the different groups (a level of 0.05 was used as the threshold for statistical significance). A Duncan test was used for post hoc comparisons when the ANOVA result was statistically significant.

\section{Results}

\subsection{Atherosclerosis Risk Factors}

The atherosclerotic risk factors, which comprise blood lipid profiles, blood glucose, and BMI of all the three groups of monkeys, have been previously reported [9]. The results for the nine euthanized monkeys included in the current study are provided in Table 2, showing that LDL but not TG was increased with the response to cholesterol (hypo, intermediate, and hyper-responsive groups). The value of TG and Glu were highest in the intermediate group compared to the other groups, while BMI was the smallest. However, the BMI of monkeys from all the groups was under $30 \mathrm{~kg} / \mathrm{m}^{2}$, indicating that they did not develop obesity. The blood glucose of monkeys in all of the groups was also in normal ranges.

Table 2. Atherosclerosis factors of nine monkeys fed an IPB-1 atherogenic diet showing differences in responses between the three groups.

\begin{tabular}{cccc}
\hline Parameter & Hyporesponsive & Intermediately Responsive & Hyper-Responsive \\
\hline TPC (mg/dL) & $158.15 \pm 9.25^{\mathrm{a}}$ & $335.56 \pm 24.63^{\mathrm{b}}$ & $478.94 \pm 17.57^{\mathrm{c}}$ \\
HDL (mg/dL) & $56.33 \pm 2.73^{\mathrm{c}}$ & $32.61 \pm 2.95^{\mathrm{b}}$ & $32.08 \pm 1.46^{\mathrm{a}}$ \\
LDL (mg/dL) & $88.18 \pm 9.83^{\mathrm{a}}$ & $202.66 \pm 22.39^{\mathrm{b}}$ & $342.70 \pm 14.91^{\mathrm{c}}$ \\
TG (mg/dL) & $40.00 \pm 2.78^{\mathrm{a}}$ & $64.33 \pm 5.020^{\mathrm{c}}$ & $42.53 \pm 3.75^{\mathrm{b}}$ \\
Glu (mg/dL) & $44.78 \pm 2.02^{\mathrm{a}}$ & $66.83 \pm 4.22^{\mathrm{c}}$ & $54.92 \pm 2.69^{\mathrm{b}}$ \\
BMI (mg/dL) & $25.21 \pm 0.73^{\mathrm{b}}$ & $22.68 \pm 0.47^{\mathrm{a}}$ & $26.34 \pm 0.41^{\mathrm{c}}$
\end{tabular}

TPC = total plasma cholesterol; HDL = high-density lipoprotein; LDL = low-density lipoprotein; TG = triglyceride $\mathrm{Glu}=$ blood glucose, $\mathrm{BMI}=$ body mass index. Different superscripts were significant at $p<0.05$ ( ${ }^{\mathrm{a}}$ lowest value followed by ${ }^{b, c}$ to compare between hypo-, intermediately, and hyper-responsive groups in a row).

\subsection{Plaque Severity}

The monkeys receiving the IPB-1 atherogenic diet displayed different grades of atherosclerosis in the CCA and BIF (Figure 1). Advanced atherosclerosis plaques, grades IV and V, were presented by six animals out of nine animals, and initial atherosclerosis lesions (grade I-III) were presented by three out of nine animals. In the hyporesponsive group, atherosclerosis grade II was most common plaque observed on the CCA. In the intermediately responsive group, $66.7 \%$ monkeys had grade IV and $33.3 \%$ had grade V. Additionally, in the hyper-responsive group, atherosclerosis occurred mostly at grade V. These findings indicate that the hyper-responsive group presents the most severe grades for atherosclerotic lesions on the CCA compared to the other groups.

On the BIF, a maximum grade of III was observed in the hyporesponsive group. In the intermediately responsive group, 33.3\% of the animals had a grade of IV, and 66.7 had a grade of V. In the hyper-responsive group, all the animals displayed a grade of V. None of the CCA and BIF sections showed an atherosclerosis lesion grade of VI. These results indicate that the atherosclerosis grade induced by IPB-1 atherogenic diet in the Indonesian cynomolgus monkey is more severe on the BIF compared to the CCA. 

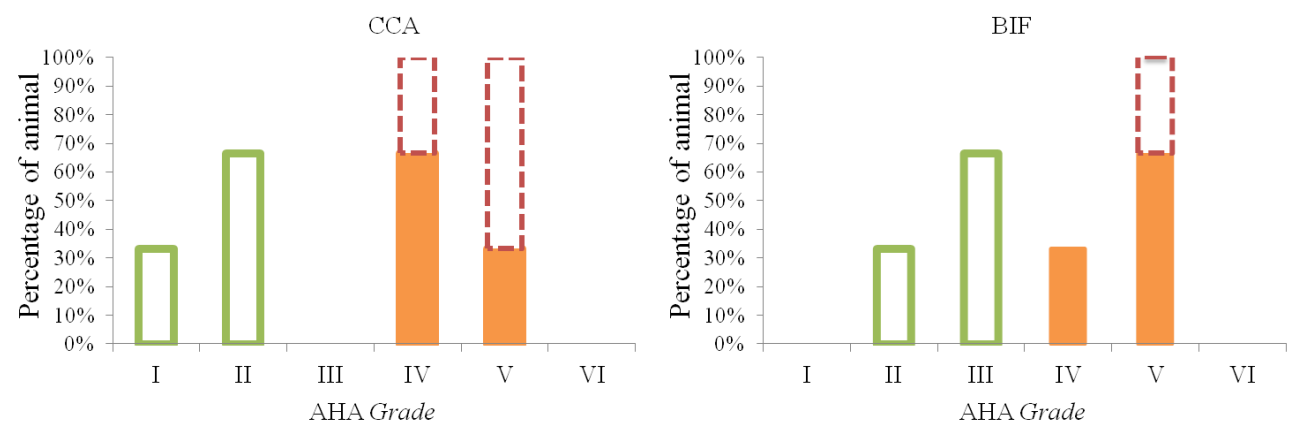

Figure 1. Different grades of atherosclerotic lesion on the common carotid arteries (CCA) and carotid bifurcation (BIF). In the hyporesponsive group ( $\square$ ), lesion grades I and II occurred on the CCA, whereas lesion grades II and III were found on the BIF. Lesion grades IV and V were observed in the intermediate group ( $(-)$ and hyper-responsive group ("). Grade V was more prevalence on the BIF compared to the CCA.

The different histopathological stages of the atherosclerosis lesion on the carotid artery of the treated monkeys are shown in Figure 2, while photomicrographs of the CCA and BIF arteries from hypo-, intermediately, and hyper-responsive group monkeys are provided in Figure 3. The carotid artery wall consists of three layers, namely the tunica intima, media, and adventitia. The normal carotid artery is characterized by a thin intimal layer and a thick tunica media that comprise numerous elastic fibers (Figure 2A). At the beginning of atherogenesis, there is a recruitment of macrophages and an increased thickness of the intima (Figure 2B). The lesion's progression continues to thicken the tunica intima of the artery by increasing foam cells (derived from macrophages), but also extracellular matrix content, and smooth muscle proliferation (called pre-atheroma) (Figure 2C). The advanced lesions are shown in Figure 2D-F. Necrosis, a fibrous cap, and calcification can be observed in the advanced lesions. Figure 3 shows that increasing the cholesterol responsiveness to the IPB-1 atherogenic diet exacerbated the formation of plaques in the carotid artery.
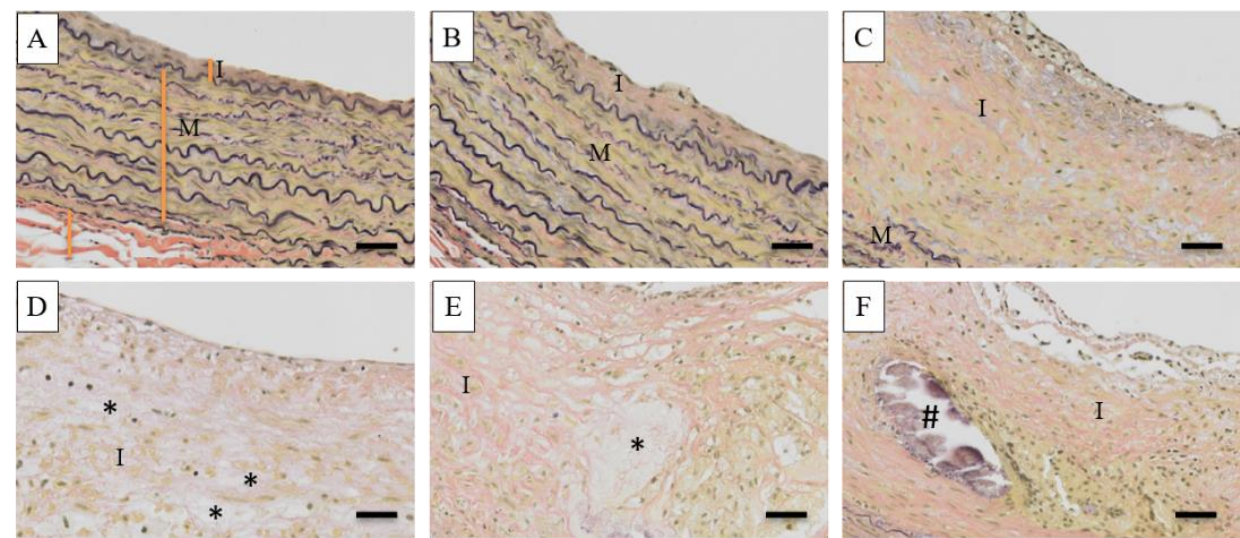

Figure 2. Histopathological stage of the atherosclerotic lesion in the carotid artery (Verhoef Van Giesson staining). (A) Normal artery showing a thin tunica intima (I) and thick tunica media (M). $(B, C)$ The initial stage of the atherosclerotic lesion shows infiltration of macrophages, as well as increasing foam cells and smooth muscle. (D) Atheroma displays thickening of the intima with a few spots of necrosis. (E,F) Fibroatheroma shows a fibrous cap, the core of the necrosis ${ }^{*}$ ), and calcification (\#). Scale bars $=20 \mu \mathrm{m}$. 


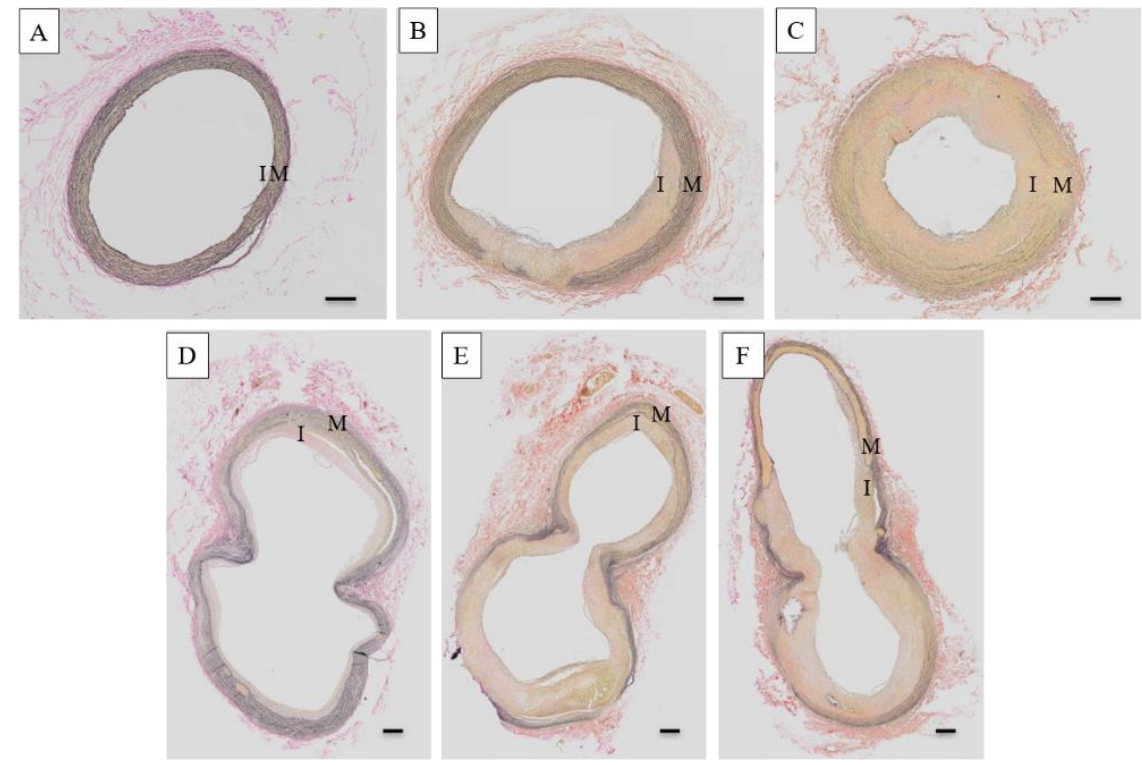

Figure 3. The atherosclerotic lesions in the common carotid arteries (CCA) (A-C) and bifurcation (BIF) (D-F) are increased with the response to cholesterol. Formation of atherosclerosis lesion increased the intimal (I) thickness and reduced elastic fibers in the media (M). No lesion was observed in the CCA of the hyper-responsive group (A). An eccentric lesion was developed in the CCA of the intermediate group (B), while a concentric lesion was developed in the CCA of the hyper-responsive group (C). A fatty streak lesion was noted in the BIF of the hyporesponsive group (D), whereas a fibroatheroma was found in the carotid bifurcation (BIF) of the intermediately and hyper-responsive groups (E,F). The most severe lesion in the CCA and BIF was observed in the hyper-responsive group $(\mathbf{C}, \mathbf{F})$. Scale bars $=200 \mu \mathrm{m}$.

\subsection{Plaque Extent}

For the CCA, monkeys receiving an IPB-1 atherogenic diet had a mean IELL of $6.72 \pm 0.10 \mathrm{~mm}$, IA of $0.45 \pm 0.06 \mathrm{~mm}^{2}$, and MXIT of $0.18 \pm 0.018 \mathrm{~mm}$. For the BIF, the mean IELL was of $13.93 \pm 0.37 \mathrm{~mm}$, IA of $2.23 \pm 0.22 \mathrm{~mm}^{2}$, and MXIT of $0.46 \pm 0.042 \mathrm{~mm}$. The differences in IELL, IA, and MXIT for three groups of responsiveness are summarized in Table 3.

Table 3. Plaque extent in the carotid artery induced by IPB-1 atherogenic diet.

\begin{tabular}{ccccc}
\hline Artery & Parameters & Hyporesponsive & Intermediately Responsive & Hyper-Responsive \\
\hline \multirow{4}{*}{ CCA } & IELL $(\mathrm{mm})$ & $6.291 \pm 0.123^{\mathrm{a}}$ & $6.828 \pm 0.128^{\mathrm{b}}$ & $7.035 \pm 0.225^{\mathrm{b}}$ \\
& IA $\left(\mathrm{mm}^{2}\right)$ & $0.045 \pm 0.007^{\mathrm{a}}$ & $0.473 \pm 0.053^{\mathrm{b}}$ & $0.821 \pm 0.109^{\mathrm{c}}$ \\
& MXIT (mm) & $0.034 \pm 0.006^{\mathrm{a}}$ & $0.216 \pm 0.019^{\mathrm{b}}$ & $0.305 \pm 0.020^{\mathrm{c}}$ \\
\multirow{3}{*}{ BIF } & IELL (mm) & $13.959 \pm 0.138^{\mathrm{a}}$ & $13.630 \pm 0.460^{\mathrm{a}}$ & $14.212 \pm 1.067^{\mathrm{a}}$ \\
& IA $\left(\mathrm{mm}^{2}\right)$ & $1.266 \pm 0.302^{\mathrm{a}}$ & $2.527 \pm 0.283^{\mathrm{b}}$ & $2.920 \pm 0.201^{\mathrm{b}}$ \\
& MXIT $(\mathrm{mm})$ & $0.307 \pm 0.074^{\mathrm{a}}$ & $0.486 \pm 0.051^{\mathrm{b}}$ & $0.597 \pm 0.034^{\mathrm{b}}$ \\
\hline
\end{tabular}

CCA = common carotid artery; BIF = carotid bifurcation; IELL = internal elastic lamina length; IA = intimal area MXIT $=$ maximum intimal thickness. Different superscripts were significant at $p<0.05 .{ }^{\text {a }}$ lowest value followed by superscripts $b, c$ to compare between hypo-, intermediately, and hyper-responsive groups in a row.

From the CCA data presented in Table 2, the IELL of the hyporesponsive group was the smallest compared to the other groups. However, the IELL did not differ between the intermediately and hyper-responsive groups $(p=0.382)$, indicating that IELL increased in the intermediately and hyper-responsive groups. The IA of the hyper-responsive group was the highest one, almost 20 times the IA of the hyporesponsive group. The smallest IA was found in the hyporesponsive group, while the IA of the intermediate group was between the hypo- and hyper-responsive groups. Similar findings were observed for MXIT, showing thicker plaques formed with higher responses to cholesterol. 
For the BIF, there was no significant difference in IELL among the groups, indicating that the responsiveness and formation of lesions did not affect this parameter. Table 2 also shows that the smallest value for both IA and MXIT on BIF was in the hyporesponsive group. No significant difference was observed in the intermediately and hyper-responsive groups for IA and MXIT, suggesting that the intermediately responsive group was able to form an atherosclerotic lesion on BIF, similarly to the hyper-responsive group.

\subsection{Immunohistochemical Staining for CD68}

The inflammatory marker CD68, which labels macrophages, was examined in this study. The percentages of CD68 staining of carotid arteries from macaques fed with the atherogenic IPB-1 are summarized in Table 4. For the CCA, approximately 1\% of the examined arterial slides had a positive reaction for CD68 in the hyporesponsive group, but this proportion increased to $30 \%$ in the hyper-responsive group. For the BIF, positive CD68 was lowest in the hyporesponsive group, followed by the intermediately and the hyporesponsive groups. CD68-positive staining in the hyper-responsive group was found in approximately $60 \%$ of the arterial slides, showing less abundant macrophages in the CCA compared to the BIF. Photomicrographs of the carotid artery stained with IHC for CD68 are shown in Figure 4.

Table 4. Mean percentages of CD68-positive (\%) staining on the carotid artery.

\begin{tabular}{cccc}
\hline Artery & Hyporesponsive & Intermediately Responsive & Hyper-Responsive \\
\hline CCA right & $1.94 \pm 1.11^{\mathrm{a}}$ & $10.15 \pm 1.97^{\mathrm{b}}$ & $29.95 \pm 4.25^{\mathrm{c}}$ \\
CCA left & $1.08 \pm 0.25^{\mathrm{a}}$ & $14.26 \pm 2.79^{\mathrm{b}}$ & $33.35 \pm 4.40^{\mathrm{c}}$ \\
BIF right & $10.70 \pm 4.01^{\mathrm{a}}$ & $46.47 \pm 5.64^{\mathrm{b}}$ & $50.09 \pm 6.25^{\mathrm{b}}$ \\
BIF left & $16.67 \pm 4.83^{\mathrm{a}}$ & $44,10 \pm 6.23^{\mathrm{b}}$ & $60.89 \pm 7.87^{\mathrm{c}}$ \\
\hline
\end{tabular}

$\overline{\mathrm{CCA}}=$ common carotid artery; BIF = carotid bifurcation. Different superscripts were significant at $p<0.05$. ${ }^{a}$ lowest value followed by superscripts ${ }^{b, c}$ to compare between hypo-, intermediately, and hyper-responsive groups in a row.

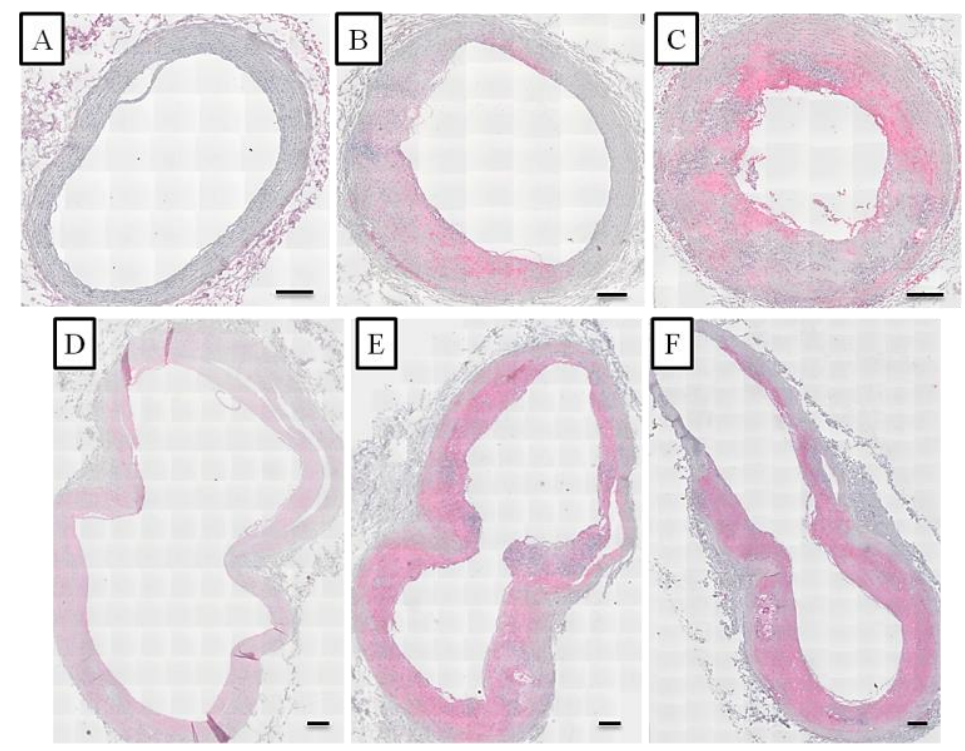

Figure 4. Immunohistochemical results of CD68 staining on the CCA (A-C) and BIF (D-F). The pink color indicates the positive CD68 visualized using vector red. The hyporesponsive group (A,D) had less prevalent CD68 staining, whereas the intermediately $(\mathbf{B}, \mathbf{E})$ and hyper-responsive groups $(\mathbf{C}, \mathbf{F})$ had the most prevalent CD68 positive staining. Scale bars $=200 \mu \mathrm{m}$.

The staining for CD68 in the carotid artery of cynomolgus monkeys receiving the IPB-1 atherogenic diet had different distributions (Figure 5). Providing information on CD68 distribution supports the determination of arterial inflammation. Figure 5 shows no 
positive CD68 staining in the normal artery. In fatty streak lesions, positive CD68 was found in some areas at the top of the tunica intima, contrary to the tunica media. In atheroma and fibroatheroma lesions, macrophages were found at the surface of the intima, around the necrotic core, and at the "shoulder" or border area between the lesion and normal area. This result indicates that macrophages can enter the healthy tissue to expand the atherosclerosis lesion.
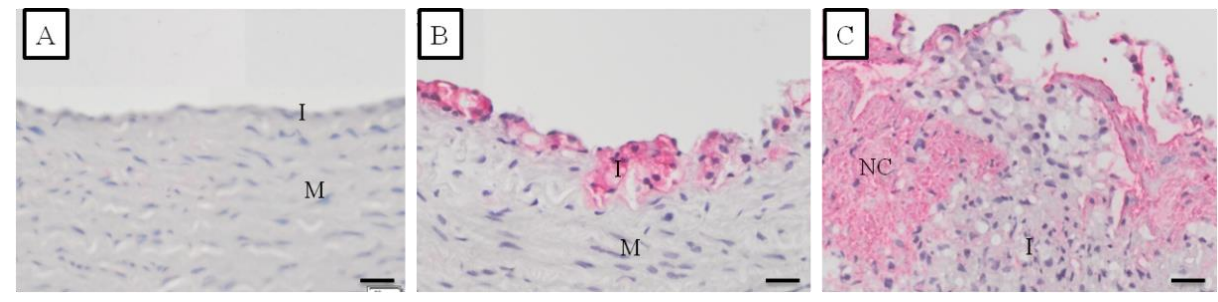

Figure 5. Distribution of CD68 staining at various stages of the atherosclerotic lesion formation in the carotid artery of the monkeys included in this study as detected by immunohistochemical (IHC) staining. The pink color indicated the positive CD68 visualized using vector red. $(A)=$ normal artery; (B) = Fatty streak lesion (AHA grade II); (C) = Fibroatheroma lesion (AHA grade IV). I = tunica intima; $\mathrm{M}=$ tunica media; $\mathrm{NC}=$ necrotic core. Scale bars $=20 \mu \mathrm{m}$.

\section{Discussion}

The development of atherosclerotic lesions is influenced by many factors, such as genetics, age, sex, hypercholesterolemia, diabetes, cigarette smoking, and stress [22,23]. The IPB-1 atherogenic diet-induced atherosclerosis risk factors of hypercholesterolemia. Different groups (hypo-, intermediate, and hyper-responsive) had different blood lipid profiles (LDL, HDL, TG), Glu, and BMI. We found that LDL but not HDL increased with the response to cholesterol. The IPB-1 atherogenic diet contains egg yolks that can increase LDL [24] and high levels of carbohydrates that can decrease HDL [25]. Hyporesponsive monkeys in this study displayed the lowest TPC, LDL, TG, and Glu levels. A similar phenomenon was reported previously in cynomolgus monkeys receiving a Western diet [14]. Recently, in Indonesian cynomolgus monkeys, genetic diversity in the $3^{\prime} \mathrm{UTR}$ of LDLR gene was associated with low blood LDL levels [26] resulting in insufficient atherosclerotic lesions.

In this study, we observed an intermediately responsive group, whereas the Western diet only induced hypo- and hyper-responsive groups previously. The IPB-1 atherogenic diet has a moderate level of cholesterol, which aims to prevent death during a two-year period of treatment. Long-term feeding and very high TPC induced by the Western diet were reported to result in depression among cynomolgus monkeys [15]. Nevertheless, consumption of IPB-1 atherogenic diet for two years was able to induce advanced atherosclerosis lesions in six out of nine monkeys in the intermediately and hyper-responsive groups. This indicates that the IPB-1 diet can be used for long-term atherosclerosis research investigating treatments for atherosclerosis as well as the regression of atherosclerotic lesions, which requires advanced lesions as observed in this non-human primate model.

The atherosclerotic lesions observed in this study histologically showed the formation of foam cells, necrotic core, fibrous cap, and calcification. However, we did not find evidence for the formation of cholesterol cleft, thin cap, and rupture. During the treatment, we maintained cholesterol supply for the monkeys around $0.28-0.29 \mathrm{mg}$ chol/Cal, which was lower than Western atherogenic diets comprising $0.35 \mathrm{mg}$ chol/Cal. The reduced level of cholesterol could have prevented its interaction with complex atheroma lesions. Lowering dietary cholesterol contents by changing cholesterol intake may reduce the atherosclerosis risk of coronary heart disease considerably in highly responsible individuals [27]. These findings indicate that a IPB-1 atherogenic diet for two years cannot be used to induce a cynomolgus monkey model of full stenosis and atherosclerotic rupture.

The atherosclerosis lesions induced by the IPB-1 atherogenic diet in Indonesian cynomolgus monkeys were also more severe on BIF compared to CCA. Plaque sever- 
ity and plaque extent of intermediately and hyper-responsive monkeys at the BIF are similar. This indicates that a slight increase in TPC could present the same risk of plaque formation on BIF. Severe atherosclerosis lesions on BIF are caused by complex fluid flow mechanics and geometric factors. High fluid flow raises the endothelial dysfunction of the artery and accelerates the atherogenesis on BIF [28,29]. Sinus enlargement of $>1.2$ times the CCA branch diameter is the most significant geometric risk factor for developing atherosclerosis in human BIF [30].

Plaque extent (IA and MXIT) on the CCA was lowest in the hyporesponsive group followed by the intermediately and hyper-responsive groups. Hypercholesterolemia caused by IPB-1 atherogenic diet thickens the tunica intima. Thickening of the tunica intima initiated by trapping and oxidation of LDL stimulates the endothelium to produce adhesion molecules and chemokines [31,32]. These inflammatory processes recruit specific infiltrating and proliferating cells, such as macrophages, lymphocytes, and smooth muscle cells in the tunica intima [8]. Consequently, raising cholesterol levels, especially LDL-cholesterol levels, increases the IA and MXIT of the artery.

Macrophages play important roles in the initiation of lesions, lesion expansion, and severe lesion formation, but also in the resolution and regression of atherosclerotic lesions [11]. The inflammation marker CD68 allows the visualization of the presence of macrophage cells in the artery. Macrophages are the most prevalent cells at the beginning of atherogenesis [13]. For the CCA, CD68 staining was very rare in the hyporesponsive group and represented around $33 \%$ of artery slides in the hyper-responsive group. Increasing responsiveness increased the positive staining for CD68 in the artery. For the BIF, CD68 staining was abundant in the hyporesponsive group and reached $50 \%-60 \%$ of the artery slides in the hyper-responsive group. The high CD68 showed high inflammation, which adversely affects the severity of atherosclerosis [33].

The distribution of CD68 staining in the Indonesian cynomolgus monkey's artery was similar to previous reports in humans. The CD68 marker in the human artery is located on the surface of the tunica intima, the necrotic core, and shoulder of the lesion [34]. In the carotid artery of the monkeys examined in this study, not all foam cells showed positive staining for CD68, probably because foam cells are also represented by smooth muscle cells [35]. From the necrotic core, macrophages enter the fibrous cap and release metalloproteinase that degrade the extracellular matrix. Degradation of the matrix makes the tissue brittle and easy to rupture [36].

\section{Conclusions}

From these results, we can conclude that feeding with a local ingredient atherogenic diet for two years can induce atherosclerotic lesions on the carotid arteries of Indonesian cynomolgus monkeys. Different stages of lesions were observed, based on the responsiveness of the monkeys to cholesterol. The hyporesponsive group did not develop proper atherosclerosis lesions in carotid arteries, indicating the importance of screening monkeys before use in atherosclerotic research. The intermediately and hyper-responsive groups developed severe atherosclerotic lesions in carotid arteries, both in the CCA and BIF. The lesions formed were similar to those observed in humans and included the distribution of macrophage cells in the lesion. These macrophages were found in the early atherosclerotic lesions and continued to expand their infiltration to the healthy arterial tissue in advanced lesions. In future studies, it will be necessary not only to explore the lesion stages but also to investigate certain molecular conditions in the artery, especially coronary arteries, together with the condition of the primary organ affected by this diet.

Author Contributions: Conceptualization, D.S., D.A.A., I.H.S., S.R.L.; methodology, S.R.L., E.H., T.C.R., I.H.S.; formal analysis, S.R.L., T.C.R.; data curation, S.R.L., T.C.R., E.H.; writing-original draft preparation, S.R.L.; writing-review and editing, S.R.L., E.H., T.C.R., I.H.S.; funding acquisition, D.S., D.A.A., S.R.L., I.H.S. All authors have read and agreed to the published version of the manuscript. 
Funding: This research was funded by the Directorate General of Higher Education, The Ministry of Education and Culture of Indonesia (PMDSU grant).

Institutional Review Board Statement: All procedures involving animals were approved by the Animal Care and Use Committee from Primate Research Center-IPB University, Number PRC-14-B003.

Informed Consent Statement: Not applicable.

Data Availability Statement: The data presented in this study are available upon reasonable request to the corresponding author.

Acknowledgments: The authors would like to thank Erni Sulistiawati for helping as a certified pathologist with the necropsy procedure, all the veterinarians and technical staff from the PRC-IPB, Indonesia, together with Deborah L Golden and other staff of the Primate Research Center Wake Forest University, NC, USA for kindly helping with data collection.

Conflicts of Interest: The authors declare no conflict of interest.

\section{References}

1. Pavlin, B.I.; Schloegel, L.M.; Daszak, P. Risk of importing zoonotic diseases through wildlife trade, United States. Emerg. Infect. Dis. 2009, 15, 1721-1726. [CrossRef] [PubMed]

2. Spiegel, C.M. Primates by the numbers: The use and importation of nonhuman primates for research and testing in the United States. AV Mag. 2011, 3-4, 1-10.

3. Kanthaswamy, S.; Ng, J.; Satkoski Trask, J.; George, D.A.; Kou, A.J.; Hoffman, L.N.; Doherty, T.B.; Houghton, P.; Smith, D.G. The genetic composition of populations of cynomolgus macaques (Macaca fascicularis) used in biomedical research. J. Med. Primatol. 2013, 42, 120-131. [CrossRef] [PubMed]

4. Sirois, M. Laboratory Animal Medicine: Principles and Procedures; Elsevier: St. Louis, MO, USA, 2005.

5. Shelton, K.A.; Clarkson, T.B.; Kaplan, J.R. Nonhuman Primate Models of Atherosclerosis. In Non-Human Primate in Biomedical Research; Abee, C.R., Mansfield, K., Tardif, S., Moris, T., Eds.; Academic Press: New York, NY, USA, 2012.

6. Eudey, A.; Kumar, A.; Singh, M.; Boonratana, R. Macaca fascicularis (amended version of 2020 assessment). The IUCN Red List of Threatened Species 2021. e.T12551A204494260. Available online: https://www.iucnredlist.org/species/12551/204494260 (accessed on 20 November 2021). [CrossRef]

7. Apri Astuti, D.; Sajuthi, D.; Herawati Suparto, I.; Kaplan, J.; Appt, S.; Clarkson, T.B. The development of diets to induce atherogenic lipid profiles for cynomolgus monkeys in their country of origin. World J. Agric. Res. 2014, 2, 247-251. [CrossRef]

8. Laila, S.R.; Suparto, I.H.; Astuti, D.A.; Sajuthi, D. The chronic effect of IPB-1 atherogenic diet on obesity and hyperglycemia in cynomolgus monkeys. JPI 2017, 14, 26-30.

9. Laila, S.R.; Astuti, D.A.; Suparto, I.H.; Handharyani, E.; Sajuthi, D. Metabolic and morphometric changes in Indonesian cynomolgus monkeys (Macaca fascicularis) fed an atherogenic diet composed of locally sourced ingredients. Vet. World 2018, 11, 1609-1617. [CrossRef]

10. Libby, P. Inflammation in atherosclerosis. Nature 2002, 420, 868-874. [CrossRef] [PubMed]

11. Tabas, I.; Bornfeldt, K.E. Macrophage phenotype and function in different stages of atherosclerosis. Circ. Res. 2016, 118, 653-667. [CrossRef] [PubMed]

12. Simionescu, M.; Sima, A.V. Morphology of atherosclerotic lesions. In Inflammation and Atherosclerosis; Wick, G., Grundtman, C., Eds.; Springer: Vienna, Austria, 2012; pp. 19-37. [CrossRef]

13. Weingand, K.W. Atherosclerosis research in cynomolgus monkeys (Macaca fascicularis). Exp. Mol. Pathol. 1989, 50, 1-15. [CrossRef]

14. Clarkson, T.B.; Alexander, N.J.; Morgan, T.M. Atherosclerosis of cynomolgus monkeys hyper- and hyporesponsive to dietary cholesterol. Lack of effect of vasectomy. Arteriosclerosis 1988, 8, 488-498. [CrossRef]

15. Chilton, F.H.; Lee, T.C.; Willard, S.L.; Ivester, P.; Sergeant, S.; Register, T.C.; Shively, C.A. Depression and altered serum lipids in cynomolgus monkeys consuming a Western diet. Physiol. Behav. 2011, 104, 222-227. [CrossRef]

16. Mirzaei, H.; Di Biase, S.; Longo, V.D. Dietary interventions, cardiovascular aging, and disease: Animal models and human studies. Circ. Res. 2016, 118, 1612-1625. [CrossRef] [PubMed]

17. Stary, H.C.; Chandler, A.B.; Glagov, S.; Guyton, J.R.; Insull, W., Jr.; Rosenfeld, M.E.; Schaffer, S.A.; Schwartz, C.J.; Wagner, W.D.; Wissler, R.W. A definition of initial, fatty streak, and intermediate lesions of atherosclerosis. A report from the Committee on Vascular Lesions of the Council on Arteriosclerosis, American Heart Association. Circulation 1994, 89, 2462-2478. [CrossRef] [PubMed]

18. Stary, H.C.; Chandler, A.B.; Dinsmore, R.E.; Fuster, V.; Glagov, S.; Insull, W., Jr.; Rosenfeld, M.E.; Schwartz, C.J.; Wagner, W.D.; Wissler, R.W. A definition of advanced types of atherosclerotic lesions and a histological classification of atherosclerosis. A report from the Committee on Vascular Lesions of the Council on Arteriosclerosis, American Heart Association. Circulation 1995, 92, 1355-1374. [CrossRef] [PubMed]

19. Bobryshev, Y.V. Monocyte recruitment and foam cell formation in atherosclerosis. Micron 2006, 37, 208-222. [CrossRef] [PubMed] 
20. Register, T.C.; Appt, S.E.; Clarkson, T.B. Atherosclerosis and vascular biologic responses to estrogens: Histologic, immunohistochemical, biochemical, and molecular methods. In Estrogen Receptors: Methods and Protocols, Methods in Molecular Biology; Eyster, K.M., Ed.; Springer Science + Business Media: New York, NY, USA, 2016; Volume 1366, pp. 517-532. [CrossRef]

21. Ridolfi, R.L.; Hutchins, G.M. The relationship between coronary artery lesions and myocardial infarcts: Ulceration of atherosclerotic plaques precipitating coronary thrombosis. Am. Heart J. 1977, 93, 468-486. [CrossRef]

22. Fruchart, J.C.; Nierman, M.C.; Stroes, E.S.; Kastelein, J.J.; Duriez, P. New risk factors for atherosclerosis and patient risk assessment. Circulation 2004, 109 (Suppl. 1), III15-III19. [CrossRef]

23. Rafieian-Kopaei, M.; Setorki, M.; Doudi, M.; Baradaran, A.; Nasri, H. Atherosclerosis: Process, indicators, risk factors and new hopes. Int. J. Prev. Med. 2014, 5, 927-946.

24. Spence, J.D. Dietary cholesterol and egg yolk should be avoided by patients at risk of vascular disease. J. Transl. Int. Med. 2016, 4, 20-24. [CrossRef]

25. Sugiri, U.; Noventi, S.; Hisatome, I.; Bahrudin, U. Carbohydrate diet links to a higher risk of significant coronary artery disease in young Indonesian patients: Cardiometabolic investigation study. Biomed. Res. 2012, 23, 159-165.

26. Taher, A.; Solihin, D.D.; Sulistyani; Sajuthi, D.; Astuti, D.A. Genetic diversity within the $3^{\prime}$ UTR of the cynomolgus macaque (Macaca fascicularis) LDLR Gene. IJSBAR 2016, 26, 237-248.

27. Van den Berg, B.M.; Spaan, J.A.; Rolf, T.M.; Vink, H. Atherogenic region and diet diminish glycocalyx dimension and increase intima-to-media ratios at murine carotid artery bifurcation. Am. J. Physiol. Heart Circ. Physiol. 2006, 290, H915-H920. [CrossRef] [PubMed]

28. Motomiya, M.; Karino, T. Flow patterns in the human carotid artery bifurcation. Stroke 1984, 15, 50-56. [CrossRef] [PubMed]

29. Goubergrits, L.; Affeld, K.; Fernandez-Britto, J.; Falcon, L. Atherosclerosis in the human common carotid artery. a morphometric study of 31 specimens. Pathol. Res. Prac. 2001, 197, 803-809. [CrossRef]

30. Kratz, M. Dietary cholesterol, atherosclerosis and coronary heart disease. Handb. Exp. Pharmacol. 2005, 170, 195-213. [CrossRef]

31. Hansson, G.K.; Hermansson, A. The immune system in atherosclerosis. Nat. Immunol. 2011, 12, 204-212. [CrossRef]

32. Galkina, E.; Ley, K. Vascular adhesion molecules in atherosclerosis. Arterioscler. Thromb. Vasc. Biol 2007, 27, 2292-2301. [CrossRef]

33. Stöger, J.L.; Gijbels, M.J.; van der Velden, S.; Manca, M.; van der Loos, C.M.; Biessen, E.A.; Daemen, M.J.; Lutgens, E.; de Winther, M.P. Distribution of macrophage polarization markers in human atherosclerosis. Atherosclerosis 2012, 225, 461-468. [CrossRef]

34. Cojocaru, E.; Trandafirescu, M.; Leon, M.; Cotuţiu, C.; Foia, L. Immunohistochemical expression of anti-CD68 antibody in atherosclerotic plaque. Rom. J. Morphol. Embryol. 2012, 53, 61-66.

35. Clarke, M.C.; Littlewood, T.D.; Figg, N.; Maguire, J.J.; Davenport, A.P.; Goddard, M.; Bennett, M.R. Chronic apoptosis of vascular smooth muscle cells accelerates atherosclerosis and promotes calcification and medial degeneration. Circ. Res. 2008, 102, 1529-1538. [CrossRef]

36. Sakakura, K.; Nakano, M.; Otsuka, F.; Ladich, E.; Kolodgie, F.D.; Virmani, R. Pathophysiology of atherosclerosis plaque progression. Heart Lung Circ. 2013, 22, 399-411. [CrossRef] [PubMed] 